\title{
Integration of Odor-Induced Activity of Kenyon Cells in an Electrotonically Compact Drosophila Mushroom Body Output Neuron $(\mathrm{MBON})$
}

\author{
Omar A. Hafez* $\quad$ Benjamin Escribano ${ }^{\dagger} \quad$ Jan Pielage $^{\dagger} \quad$ Ernst Niebur*ł $^{* \ddagger}$
}

January 22, 2021

\begin{abstract}
The formation of an ecologically useful lasting memory requires that the brain has an accurate internal representation of the surrounding environment. In addition, it must have the ability to integrate a variety of different sensory stimuli and associate them with rewarding and aversive behavioral outcomes. Over the previous years, a number of studies have dissected the anatomy and elucidated some of the working principles of the Drosophila mushroom body (MB), the fly's center for learning and memory. As a consequence, we now have a functional understanding of where and how in the MB sensory stimuli converge and are associated. However, the molecular and cellular dynamics at the critical synaptic intersection for this process, the Kenyon cell-mushroom body output neuron (KC-MBON) synapse, are largely unknown. Here, we introduce a first approach to understand this integration process and the physiological changes occurring at the KC-MBON synapse during Kenyon cell (KC) activation. We use the published connectome of the Drosophila MB to construct a functional computational model of the MBON- $\alpha 3$ dendritic structure. We simulate synaptic input by individual KC-MBON synapses by current injections into precisely $(\mu m)$ identified local dendritic sections, and the input from a model population of KCs representing an odor by a spatially distributed cluster of current injections. By recording the effect of the simulated current injections on the membrane potential of the neuron, we show that the $\mathrm{MBON}-\alpha 3$ is electrotonically compact. This suggests that odor-induced MBON activity is likely governed by input strength while the positions of $\mathrm{KC}$ input synapses are largely irrelevant.
\end{abstract}

\section{Introduction}

For living organisms, interactions with the environment strongly depend on their capability to form an internal representation of the surrounding world. Storing information about potential rewards and dangers requires a constant update of such representations and it is crucial for survival. This allows the organism to adapt its future behavior with the goal of increasing rewarding interactions with the environment. In the brain, information of different stimuli can be associated through learning and then memorised over long periods of time. Recall of such associations will then lead to an adapted behavioral output.

Here, we use the Drosophila melanogaster mushroom body (MB) as a model to study the cellular basis of learning and memory. The MB is required for acquisition and recall of associative olfactory memories[12, 24, 31, 32, 37, 51]. Among the advantages of this structure is that the number of neurons in the MB is relatively small and that, in Drosophila, many of these neurons are genetically accessible through a large variety of tools that allow visualisation and neuronal manipulation of neuronal subpopulations [13, 16, 23, 25, 27, 30, 36, 39, 44, 48]. The MB consists of about 2000 Kenyon cells (KCs) per hemisphere that receive stochastic innervation by projection neurons (PNs) from the antennal lobes $[29,46]$. The antennal lobes, in turn, receive direct input from the olfactory receptor neurons (ORNs) of the antennae [52]. Stochastic innervation of KCs therefore generates a random and sparse representation of odors in the MB $[7,21,50]$. The somata of all KCs are clustered in the posterior brain and project their axons into the MB calyx, the horizontal and vertical lobes of the MB [11, 26, 28, 47]. KCs then innervate 22 mushroom body output neurons (MBONs) per hemisphere in a highly compartmentalised

\footnotetext{
*Zanvyl Krieger Mind/Brain Institute, Johns Hopkins University, Baltimore, MD 21218, USA

${ }^{\dagger}$ Division of Neurobiology and Zoology, University of Kaiserslautern, Kaiserslautern, Germany

¥Solomon Snyder Department of Neuroscience, Johns Hopkins University, Baltimore, MD 21218
} 
manner [3]. Current data supports a balance model, in which the net output activity of the MBONs defines the behavior of the fly when it encounters a previously conditioned odor $[2,3,9]$.

Information regarding reward or punishment of an odor is provided by dopaminergic neurons (DANs) [6] that innervate the $\mathrm{KC}-\mathrm{MBON}$ synapse that also respect the compartmentalised nature of the MB [10]. Coincident activation of a $\mathrm{KC}$ and local release of dopamine is thought to selectively depress KC-MBON synapses resulting in modified $\mathrm{MBON}$ activity $[4,35,40,42]$ and, as a consequence, altered behavior of the fly [19]. Therefore, the output behavior to the conditioned odor will be changed [49]. Despite the accumulated knowledge of the structure of the $\mathrm{MB}$ and the functional model of its influence on fly behavior, the molecular and cellular processes at the KC-MBON synapse inducing and maintaining decreased activation of the MBON remain largely unknown.

Importantly, the recent complete electron-microscopic (EM) reconstruction of the Drosophila MB [45] and the entire fly brain [53] provided precise information of the anatomical structure of KC, DAN and MBONs and their synaptic interactions. In addition, we recently developed a novel tool to selectively mark, and manipulate the activity of, individual KCs that store associative aversive olfactory long-term memory [43]. This tool enables us to directly access KCs and to evaluate synaptic connectivity changes to the MBONs that are relevant to modulate behavioral changes.

In the present study, we combine our knowledge of KC-based storage of long-term memories [43] with the precise anatomical data provided by EM-studies $[14,45]$ to determine the nature of KC-MBON interaction. As a starting point we use the MBON- $\alpha 3$ neuron which receives 12,770 input synapses from KCs. We perform computational simulations to determine the relevance of individual synaptic input along the dendritic tree to provide an estimate regarding the number of synapses and KCs necessary to evoke MBON activation. Our study provides first evidence that MBON- $\alpha 3$ is electrotonically compact and demonstrates that few KCs may be sufficient to evoke reliable MBON activation.

\section{Results}

In this study we make use of the known dendritic structure of the MBON- $\alpha 3$ to generate a computational model of the effect of input $\mathrm{KC}$ synapses on the membrane potential in this neuron. Based on electrophysiological data, Cassenaer and Laurent [8] have suggested that Drosophila MBON neurons are electrotonically compact. One purpose of our computational study is to determine to what extent this is the case for one specific neuron, $\mathrm{MBON}-\alpha 3$. Given the observed unipolar morphology of an MBON, we assumed passive (linear) cable-theory to model the neuron's functionality. In general, it is not yet understood to what extent nonlinear currents occur in the dendritic tree of MBONs. Therefore, we will test to what extent the neuron is functional in their complete absence. We consider this, on the one hand, as a necessary baseline condition that can be extended if either experimental evidence becomes available that requires revision of this hypothesis, or if the assumption of passive dendrites is demonstrably not compatible with the functionality of this neuron. On the other hand, it is the simplest assumption that can be made for this structure, and if the computational results show that the neuron can fulfill its function under this assumption, an argument can be made that no more complex mechanisms are required. This simple model is then preferable over more complex ones by the principle of "Occam's Razor."

If the neuron is electrotonically compact, small current injections made at different locations along its dendritic tree will have identical effects on the membrane potential at the neuron's proximal neurite. We, furthermore, hypothesize that a small number of injected currents with the characteristics of typical excitatory synaptic inputs, representing the typical input pattern received from KCs, would be sufficient to excite the MBON by depolarizing its proximal neurite past the threshold for firing action potentials. This hypothesis is motivated by the fact that, to compensate for the small number of active synapses for any one odor, the synapses between KCs and the MBONs are particularly strong, with an average excitatory post-synaptic potential (EPSP) of $1.58 \mathrm{mV}[8]$.

Using the NEURON environment [20], we simulated such small current injections at different locations along the MBON- $\alpha 3$ dendritic tree. To anticipate our results, our data provide first evidence that MBON$\alpha 3$ is electrotonically compact. Furthermore it provides insight into the integration of learned olfactory information at MBON dendritic trees.

We first (Section 2.1) show examples of excitations at randomly selected synapses. Subsequently, we systematically excite selected segments (Section 2.3) and patterns of segments (Section 2.2). Note that stimulation currents in Section 2.1 have a duration of $100 \mathrm{~ms}$ (and a correspondingly low current to generate EPSPs compatible with experimental observations). This is meant only as an illustration and to probe the neuron's behavior with a simple stimulation protocol that allows us to compare the shape of the voltage excursion over a longer time course (Fig. 1H below). Data in Section 2.3 and 2.2 are obtained with a stimulation pattern where each dendritic segment (= model synapse) is excited by a square current 


\begin{tabular}{|c|c|c|c|c|c|}
\hline Fig. & $\mathrm{I}[\mathrm{nA}]$ & Pulse length $[\mathrm{ms}]$ & \# synapses & Recording site & max V $[\mathrm{mV}]$ \\
\hline \hline 1C,E & 0.0017 & 100 & 1 & dendrite & $-58.5(\mathrm{C}),-58.3(\mathrm{E})$ \\
\hline 1D,F & 0.0017 & 100 & 1 & proximal neurite & -59 \\
\hline 3B,C & 0.02 & 5 & 1 & dendrite & $\approx[-55,-45]$ \\
\hline 3D,E & 0.02 & 5 & 1 & proximal neurite & $\approx[-58,-57]$ \\
\hline 2B,C & 0.02 & 5 & 25 & proximal neurite & $\approx[3,6]$ \\
\hline
\end{tabular}

Table 1: Overview of Results figures.

\begin{tabular}{|c|c|c|c|}
\hline Variable & Description & Assigned Value & Notes \\
\hline \multicolumn{4}{|c|}{ Electrophysiologic Properties } \\
\hline nseg & Number of segments in section & 1 & \\
\hline $\mathrm{Ra}$ & Cytoplasmic resistivity $[\Omega \times \mathrm{cm}]$ & 260 & ref. [18] \\
\hline $\mathrm{cm}$ & Specific membrane capacitance $\left[\mu \mathrm{F} / \mathrm{cm}^{2}\right]$ & 0.9 & ref. [17] \\
\hline \multicolumn{4}{|c|}{ Passive Channel Properties } \\
\hline g_pas & Passive conductance $\left[\mathrm{S} / \mathrm{cm}^{2}\right]$ & $4.5 \times 10^{-5}$ & $\begin{array}{c}\tau=20 \times 10^{-3} \mathrm{~s}[8] \\
\text { Computed as: } \\
\tau=R_{m} C_{m} \\
g_{\text {pas }}=1 / R_{m}\end{array}$ \\
\hline e_pas & Leak reversal potential $[\mathrm{mV}]$ & -60 & ref. [5] \\
\hline
\end{tabular}

Table 2: Electrophysiological properties of the Drosophila MBON- $\alpha 3$ used in the NEURON environment. Parameter values are obtained either directly through literature search or, where necessary, calculated based on available data, as noted. The passive conductance $g_{\text {pas }}$ was computed from the cell time constant [8] and the specific membrane capacitance $C_{m}$, see Section 4.1 for details.

pulse of $5 \mathrm{~ms}$ duration. These current injections are a simple approximation to fast synaptic activation currents, like those resulting from glutamatergic $\alpha$-amino-3-hydroxy-5-methyl-4-isoxazolepropionic acid receptor (AMPA-R) type synapses. To guide the reader, Table 1 gives an overview of all Results figures.

\subsection{Time course of voltage excursions in dendrite and proximal neurite}

Simulated current was injected into one randomly selected dendritic section (\#1467; Figure 1B, C), and the resulting change in membrane potential at the proximal neurite was recorded. Before current injection, the simulation was run for $200 \mathrm{~ms}$ simulated time without input to allow the system to equilibrate, after which current with an amplitude of $1.7 p A$ was injected for ${ }^{1} 100 \mathrm{~ms}$ (Figure $1 \mathrm{G}$ ). This was sufficient to depolarize the dendritic membrane voltage in this segment by $+1.5 \mathrm{mV}$ (Figure 1C), the approximate size of a typical EPSP for a KC - MBON synapse [8]. The simulation lasted for 1,000ms in total duration. This was repeated at a different random location on the dendrite tree (\#4149; Figure 1B, E) to determine whether the location of current injection influenced the change in membrane potential at the proximal neurite (Figure 1D,F). Figure 1H shows that the voltage traces in the proximal neurite from the two excitations overlap nearly perfectly, suggesting that the location of synaptic input has very little influence on their effect on proximal neurite excitation. This is an indication that the neuron is electrotonically compact. In the following Section we will investigate how general this result is by systematically exciting a large variety of segments in the dendritic tree.

\subsection{Distributions of voltage excursions in dendrite and proximal neurite for distributed activation patterns of synapses}

It was previously established that about $5 \%$ of all Kenyon cells are activated upon stimulation by one odor [7]. Former studies have also shown that a subset of engram cells in $\approx 500 \alpha \beta$ surface neurons (a subclass of KCs) are required for memory recall $[22,43]$. Therefore we hypothesized that excitation of $5 \%$ of all $\alpha \beta$ surface neurons would represent a memory-relevant and physiologically realistic odor induced activation of an MBON. We reduced the network to one KC-MBON synapse per KC for a first simplified approach. We therefore studied the depolarization at the proximal neurite following simultaneous current

\footnotetext{
${ }^{1}$ We chose to make the current injection very long (100ms) to demonstrate the nearly perfect overlap of the proximal neurite voltages for different stimulated segments (Figure $1 \mathrm{H}$ ) even for this long duration. As mentioned, the current amplitude was adjusted to result in a realistic dendritic EPSP size.
} 
A

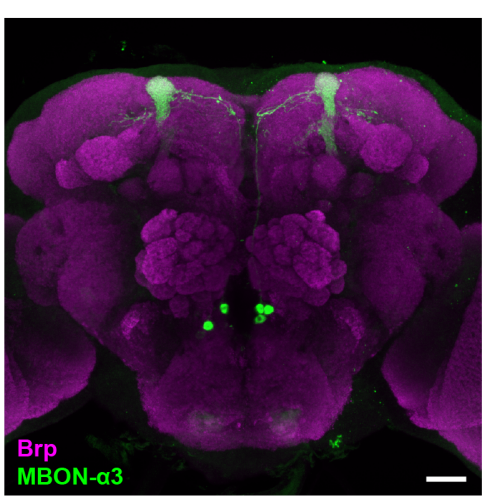

C

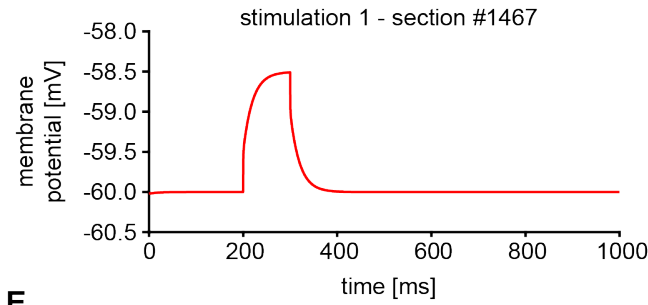

E

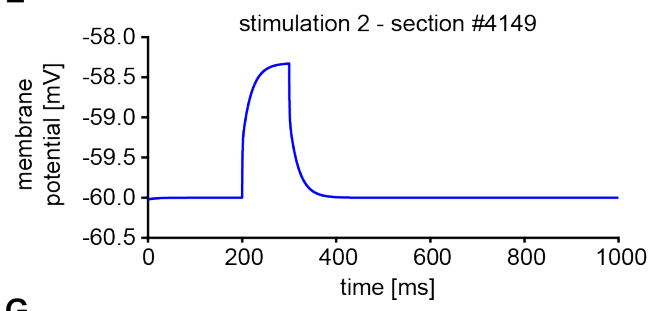

G

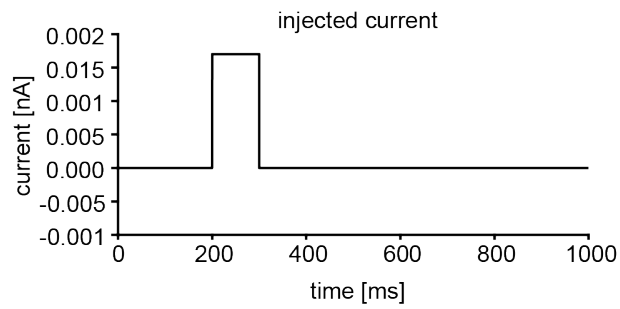

B

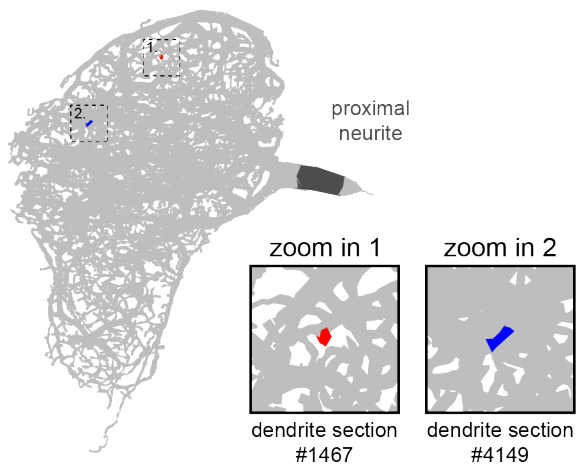

D

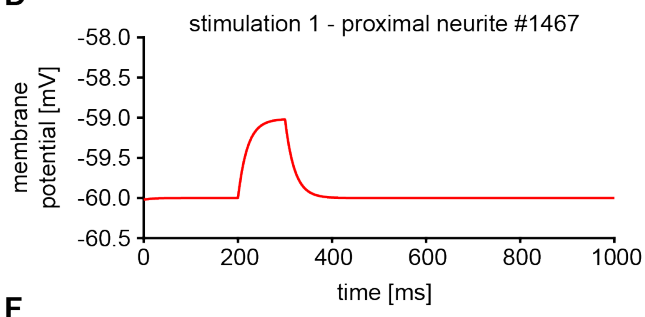

-58.0 stimulation 2 - proximal neurite \#4149

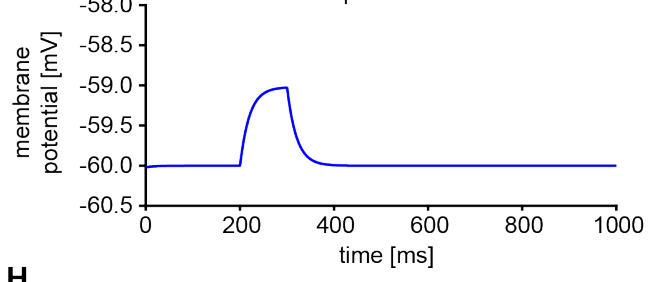

H

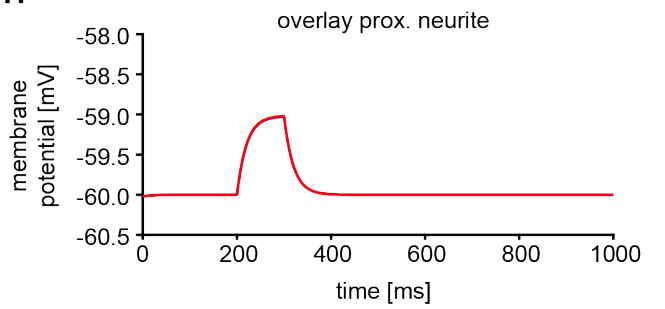

Figure 1: Constructing a computational model of MBON- $\alpha 3$. (A) Immunohistological staining of MBON$\alpha 3$ (green) and Bruchpilot (Brp) as a marker for neuronal active zones (magenta). See Section 4.2 for details. (B) Structure of the Drosophila MBON- $\alpha 3$ dendritic tree visualised by NEURON. Morphology of the dendritic arborisations as characterised by Takemura et al. [45]. The morphology of the soma and axon are not known. Magnified boxes represent stimulated dendritic segment \#1467 (red) and \#4149 (blue). The proximal neurite at the base of the dendrite is darkened for visualization. A square-pulse current (see G) was injected into either dendritic section \#1467 (C) or \#4149 (E), resulting in a depolarization of the membrane potential by approximately $+1.6 \mathrm{mV}$ at the location of current injection. The effect of these current pulses on the proximal neurite is shown in (D) for segment \#1467 and in (F) for \#4149. In both cases, it resulted in membrane potential depolarisations of about $1.0 \mathrm{mV}$. (G) Protocol of injected current with an amplitude of $1.7 p A$ for $100 \mathrm{~ms}$. (H) Overlay of membrane potentials in proximal neurite resulting from the two current injections (from D and F) shows nearly identical voltage excursions. 
pulse stimulation of 25 segments distributed over the MBON's dendritic tree. Synaptic activation was simulated in each of these segments by injected current pulses with an amplitude of $0.02 \mathrm{nA}$ and duration of $5 \mathrm{~ms}$ (same as in Section 2.3 below).

Figure 2B shows an overlay of all voltage traces for 200 trials, each with a different set of randomly selected stimulated segments. Supplementary Figure S1B,C shows the corresponding histogram of maximal voltage excursions in the proximal neurite. Peak voltages were all positive (min: $2.82 \mathrm{mV}$, max: $6.18 \mathrm{mV}$, average: $4.23 \mathrm{mV}$ ). The fact that this distribution is wider than that of the individual EPSPs at the proximal neurite (Figure 3E) may indicate that nonlinear interactions between currents become important. However, all combined EPSPs are far above spiking threshold, with a substantial "safety factor," so functionally this may be of limited relevance.

\subsection{Distributions of voltage excursions in dendrite and proximal neurite due to individual synaptic excitations}

The simulated voltage injections in Section 2.1 were applied in two randomly selected dendritic segments. Section 2.2 showed that a pattern of synaptic activation similar to what is assumed to occur in response to odor delivery to the insect reliably brings the MBON voltage above threshold. In this section we investigate the effects of current pulses injected into individual segments in the dendritic tree, and repeat this for many segments. We record the effect the injections have on the voltage of the proximal neurite as well as in the dendrite at the point of injection, and determine the voltage distributions. If the neuron is electrotonically compact, we expect the proximal neurite voltage distribution to be narrow.

Current pulses were injected systematically into segments of the dendritic tree, one at a time. We selected every fourth of the 4361 segments that NEURON divided the dendritic tree into, for a total of 1091 different segments. The segments that were stimulated are marked in red in Figure 3A. Each current pulse had a duration of $5 \mathrm{~ms}$ and an amplitude of $0.02 n A$. The amplitude was selected to obtain a dendritic EPSP in approximate agreement with the observed values [8].

A plot of all evoked dendritic EPSPs is shown in Figure 3B. The statistical distribution of the maximal depolarizations (Amplitude) is plotted in Figure 3C. The average of maximal voltage excursion is $-50.06 \mathrm{mV}$, and most EPSPs fall into a range of about $10 \mathrm{mV}$, from $-45 \mathrm{mV}$ to $-55 \mathrm{mV}$. We surmise that the range of $\approx \pm 5 \mathrm{~ms}$ for identical current injections is due to differences in local dendritic geometry.

The effect of all single synapse stimulations on the proximal neurite voltage is shown in Figure 3D and their statistical distribution in Figure 3E. The average EPSP peak at the proximal neurite was found to be $-57.40 \mathrm{mV}$. The depolarizations fall into the range $[-57.57 \mathrm{mV},-56.85 \mathrm{mV}]$, indicating a very compact structure. This range is narrower by an order of magnitude than the range of dendritic EPSPs (Figure 3B). It therefore appears that propagation along the dendritic structure evens out differences arising at the local dendritic site of activation, resulting in a "compactifying" effect. Equalization of synaptic input from different KCs may be of consequence for the representation of odorant information.

\subsection{Voltage attenuation in the dendritic tree}

The majority of voltages in the proximal neurite elicited by identical current injections is in a relatively small range of approximately $\pm 5 \mathrm{mV}$ (Figure 3D). We assumed that differences in proximal neurite voltages are due at least in part to two factors.

The first factor are the differences in the distances between the stimulation sites and proximal neurite. For the selected stimulation sites (and likely for the dendritic tree as a whole), these distances range from about $2 \mu \mathrm{m}$ to about $65 \mu \mathrm{m}$, see Supplementary Figure S2E,F. To test this hypothesis, we plot in Figure 4A the distance between stimulated segment and proximal neurite $v s$. the difference in maximal voltage in the proximal neurite and the dendrite. We find an overall positive correlation between these quantities, supporting our hypothesis.

The second factor that we hypothesized playing a role in the voltage attenuation are the differences between dendritic voltages in response to (identical) current injection due to differences in local geometries. To understand this factor better, Figure $4 \mathrm{~B}$ shows for each simulation the value of proximal neurite voltage as a function of the corresponding dendritic voltage. We observe a positive correlation between these two quantities, corroborating our second hypothesis. In this figure, we also use a color code to show the distance along the dendrite between proximal neurite and current injection site. This distance is generally inversely related to the proximal neurite voltage, as we already saw in Fig. 4A. 
A

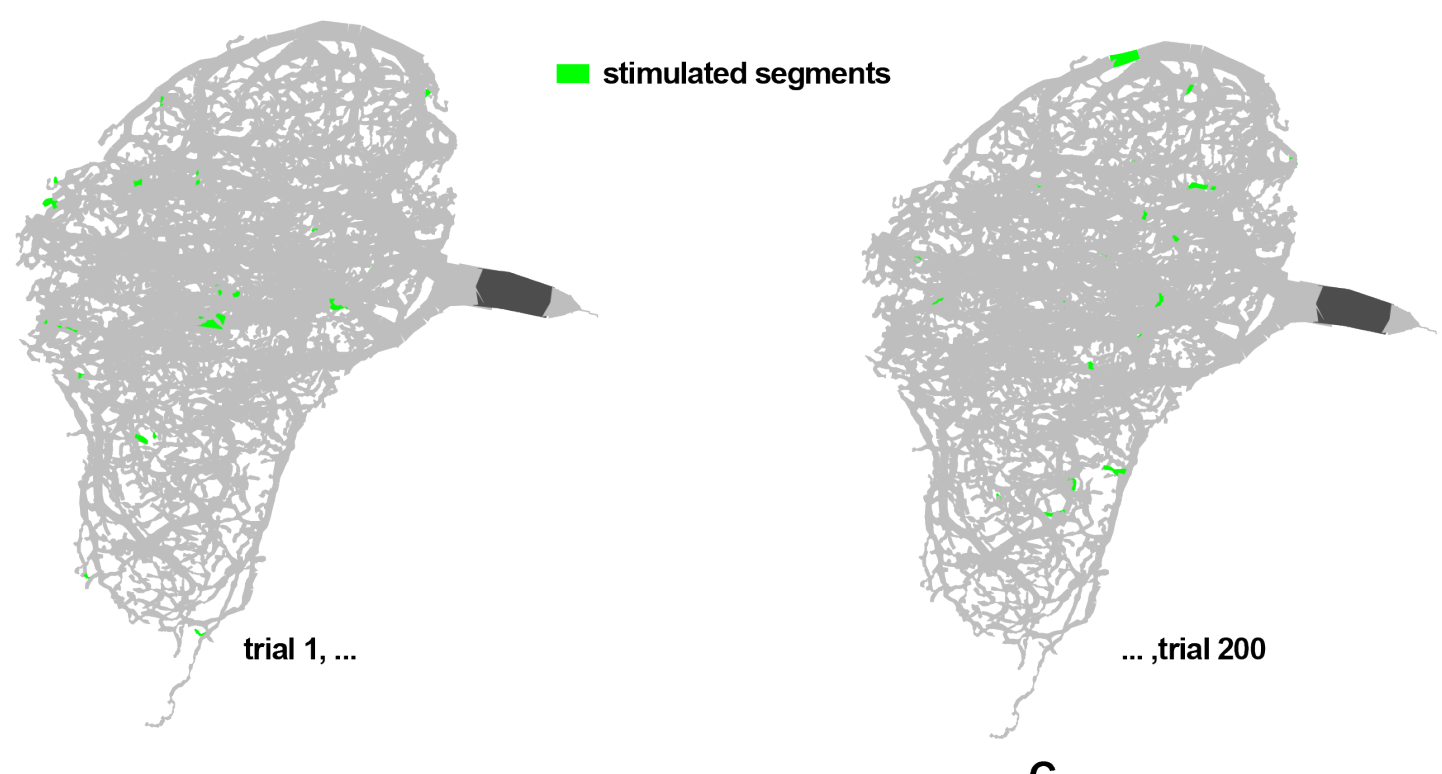

B
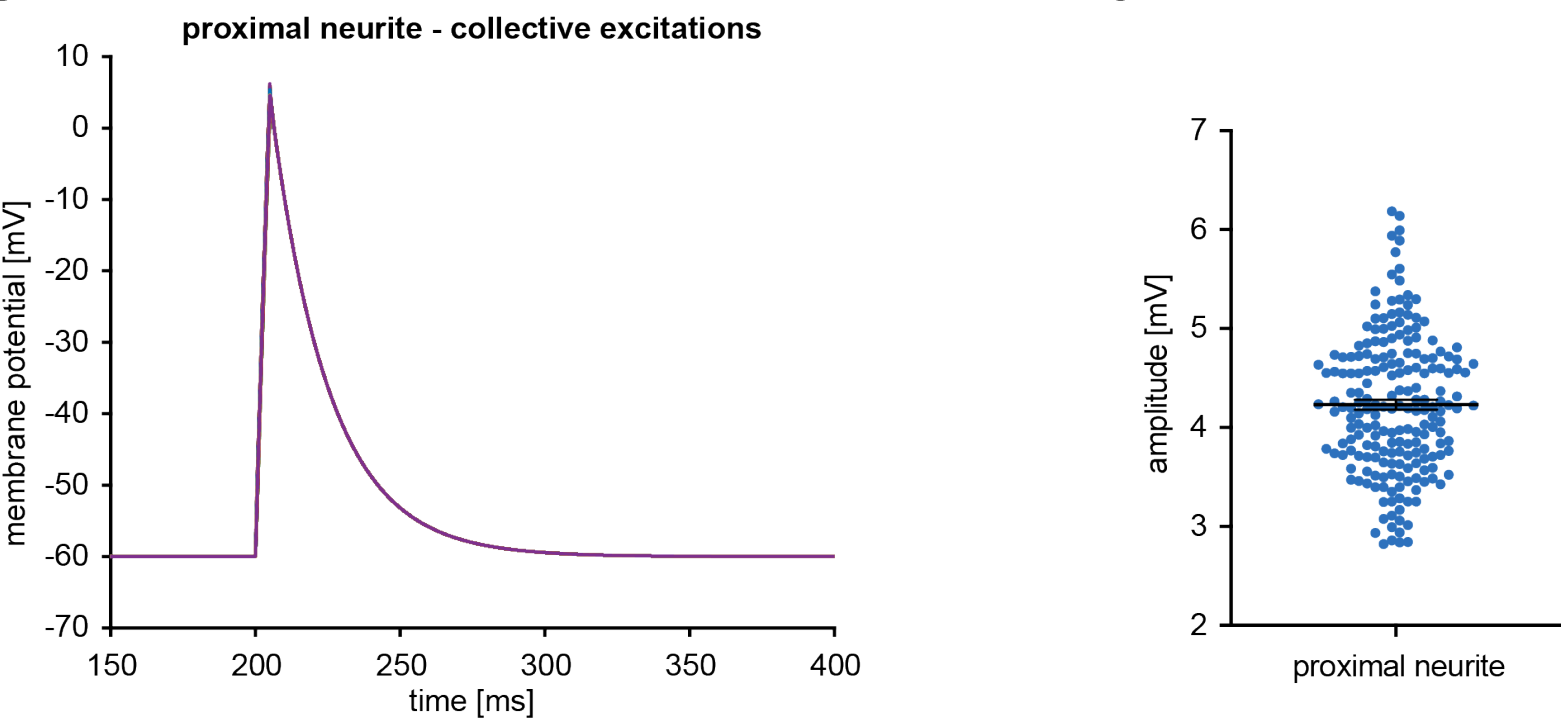

Figure 2: Simultaneous current injections of multiple sections in the MBON- $\alpha 3$ dendritic tree to mimic natural odor input. (A) In each "trial" (simulation run), 25 dendritic segments (green; many are not visible in the figure because they are behind non-stimulated segments which are shown in gray) were selected randomly and identical current pulses of $5 \mathrm{~ms}$ duration were injected simultaneously. A total of 200 trials were conducted. (B) Overlay of all 200 voltage traces in the proximal neurite. (C) Statistical distribution of all maximal voltage amplitudes in the proximal neurite with mean (dashed line). Error bars represent standard deviation (SD). 
A

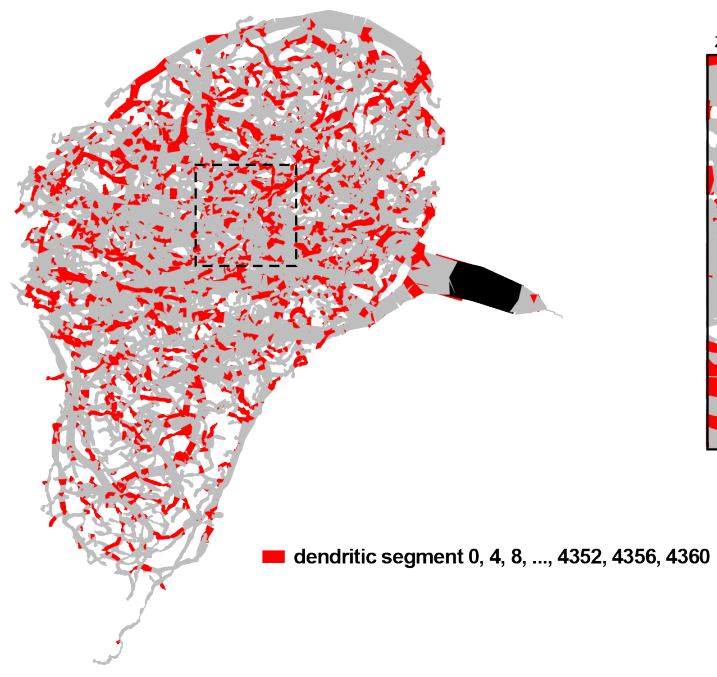

B

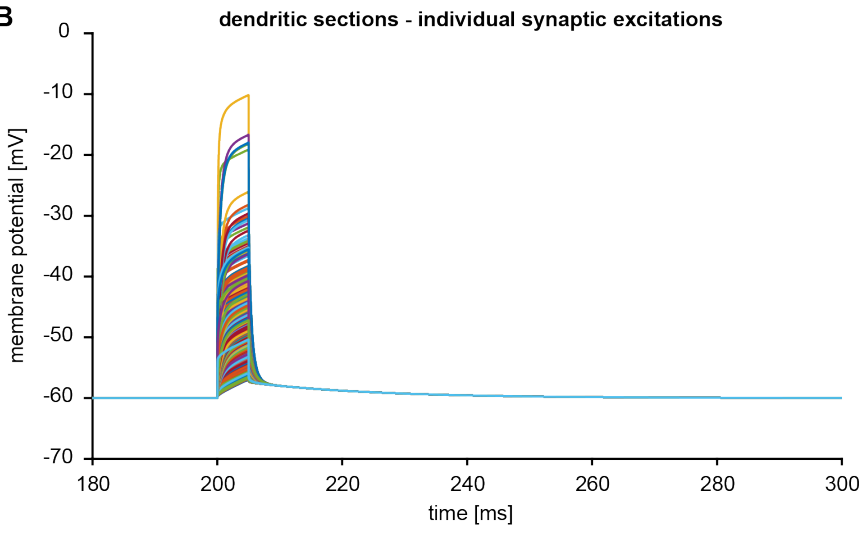

D

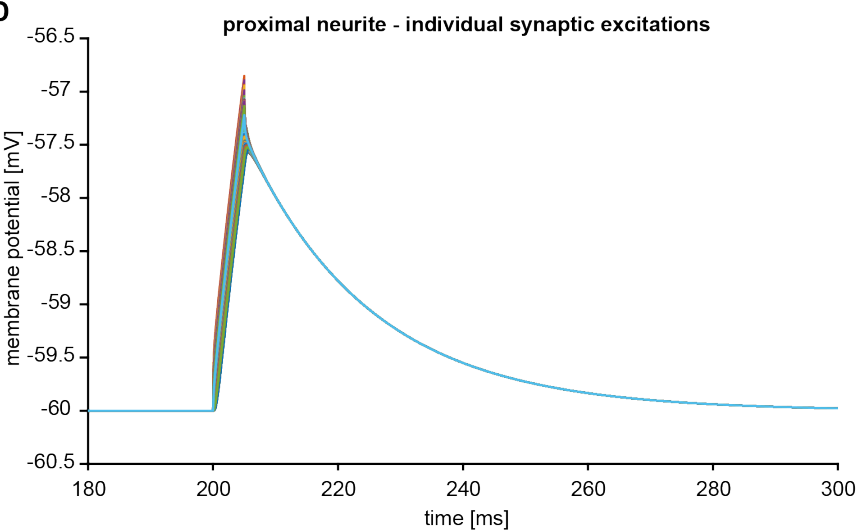

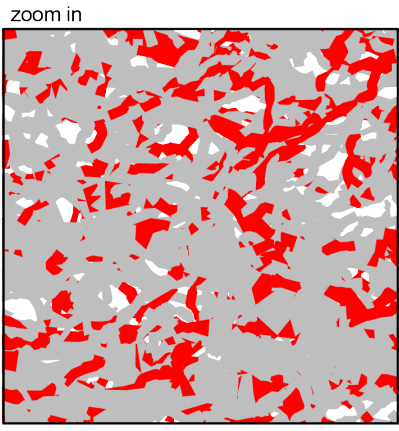

C

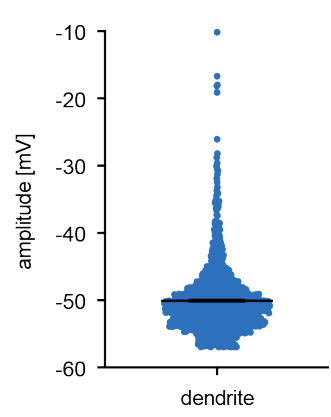

E

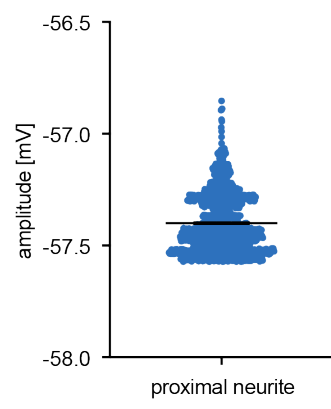

Figure 3: Segmentation and widespread activation of single dendritic segments to study electrotonic compactness. (A) For an even distribution every fourth segment of the dendritic tree (red) was selected for current injection. In total 1091 segments were stimulated. The voltage after current injection was measured in the stimulated segments themselves $(B, C)$ or in the proximal neurite (black) $(B, C)$. (B) Voltage in all activated dendritic segments. (C) Distribution of maximal voltages in all stimulated dendritic segments, with mean and SD. (D) Corresponding voltages in the proximal neurite for all activated dendritic segments. (E) Distribution of maximal voltage in the proximal neurite with mean and SD. Note the difference in vertical scales in panels $\mathrm{C}$ and $\mathrm{E}$. 
A

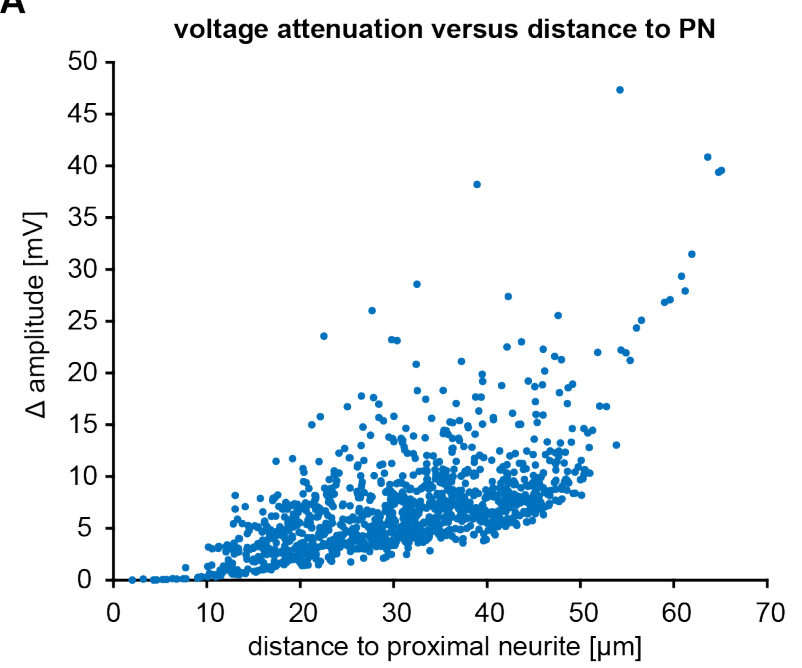

B

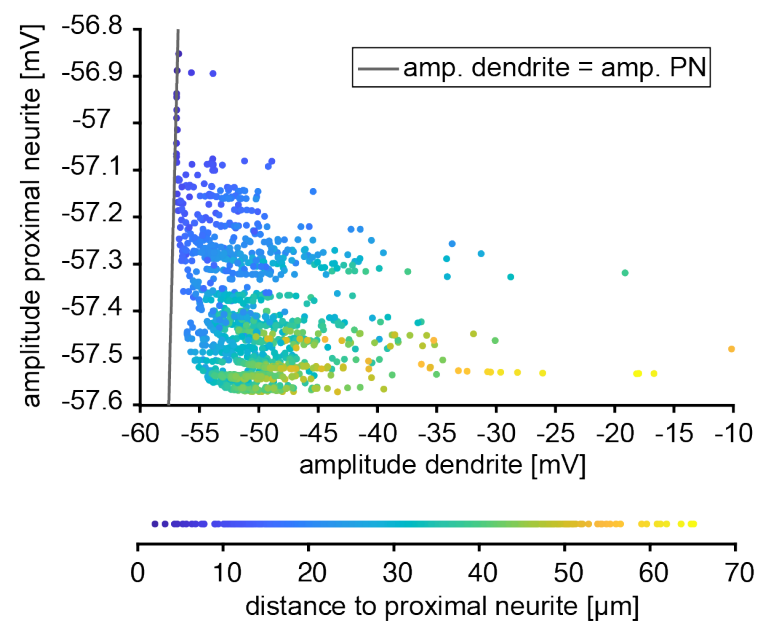

Figure 4: (A) Voltage attenuation between dendritic tree and proximal neurite. Attenuation is computed as the difference between the maximum voltage in each of the $n=1091$ stimulated dendritic segments and in the proximal neurite ( $\Delta$ Amplitude). It is plotted vs. the distance between the dendritic segment and the proximal neurite segment. (B) Voltage excursion in the proximal neurite as a function of voltage excursion in the stimulated dendritic segment. Color code represents the distance of the stimulated dendritic section from the proximal neurite.

\section{Discussion}

Our numerical simulations show that the MBON- $\alpha 3$ neuron is electrotonically compact. As a consequence, the impact a synapse has on the output of this neuron, i.e. generation of action potentials, is to a large extent independent of where on its dendritic tree the synapse is located. These simulation results are in agreement with a simple analytical estimate of the space constant of this neuron. This is obtained by assuming a non-branching cylinder with the mean diameter of the dendritic segments, $0.29 \mu \mathrm{m}$, as obtained from the morphological data from Takemura et al. [45]. We assume values for the conductance of the cytoplasm of $4.5 \times 10^{-5} \mathrm{~S} / \mathrm{cm}^{2}$ and transmembrane conductance of $260 \Omega \mathrm{cm}$. These values were obtained by Gouwens and Wilson [18] in a combined electrophysiological and computational study of antennal lobe projection neurons, another class of Drosophila central neurons. The characteristic length is then computed by linear cable theory [34] as $\lambda \approx 350 \mu m$, more than three times the maximal path length between segments of the dendritic tree and the distal end of the EM reconstruction.

\subsection{Limitations}

The results obtained from these simulations are potentially limited by the available data for the specific neuron that had been modeled. While the precise morphology has recently been characterized for the MBONs in Drosophila [45], data describing the cell's electrophysiological properties such as channel conductances and cytoplasmic resistivity have not been quantified. Therefore, to build the constructed model we were forced to assume that the MBON- $\alpha 3$ has properties similar to MBONs characterized in other insects as well as other neurons in the insect central nervous system.

Another limitation is that synaptic activation is simulated as current pulses, rather than conductance changes. This approximation is valid if interactions between synaptic currents are minimal. This may be the case here since only a very small fraction of synapses (a single one in Sections 2.1, 2.3 and 2.4, or 25 in Section 2.2, in all cases out of 12,770) is activated in the simulation. However, with the level of injected currents chosen, voltages at the proximal neurite show large deflections from rest (up to positive values) so this assumption may not be valid in all parts of the neuron. In future work we plan to go beyond this approximation, see also section 3.3.

Another potential concern is that the shape of the current injections (a square pulse) is unrealistic. It would be simple to replace it by a more realistic shape, like an $\alpha$-function but it seems unlikely that this will have a substantial effect on results.

The current simulations assume identical stimulation at each synapse. As a second step it might also be interesting to use a mix of synaptic strengths from a distribution over some range. We know that KC- 
MBON synapses are not homogeneous entities. Cassenaer and Laurent [8] showed that the physiological impact of synapses varies over a range of at least a magnitude, and Takemura et al. [45] found that the physical size of synapses varies over a considerable range. The fact that we recently observed bundles of labelled CAMEL cells after paired conditioning of flies underpins this hypothesis [43]. It is possible though unproven at this time that these physiological and morphological differences in synaptic strength are caused by their plasticity during learning. The impact of such variability on the output of the MBON is of great interest. There is also variation around the mean of measured dendritic time constants, and concomitant local variations of membrane conductances. This is another target for adding a stochastic component to the simulation.

\subsection{Deviations from physiological conditions}

In our simulation experiment, we approximated the synaptic excitation pattern generated by an odor input by simultaneous current injections in 25 locations of the MBON dendrite. However, the currently published connectome gives more profound insight into definite numbers of KC-MBON connectivity. In this study, we start with a simplified excitation pattern to establish as a proof of principle. In the future, we will take into account more details in the numbers, and detailed locations, of KC-MBON synapses.

\subsection{Model extensions}

Excitability of the MBON- $\alpha 3$ was examined by determining whether current injections along the dendritic tree could depolarize the cell past an assumed firing threshold in the spike initiation zone (SIZ; we do not know in detail where the SIZ is located and therefore used the proximal neurite as a simple approximation for it). Future work can augment this approach in several ways. First, the input current can be modeled by replicating the dynamics of the excitatory $\mathrm{KC}-\mathrm{MBON}$ synapses, beginning by incorporating into the model representations of spiking KCs releasing excitatory neurotransmitter onto receptors within postsynaptic densities located at identified KC-MBON synapses on the MBON dendrites. Additionally, active channels can be inserted into the membrane of the putative SIZ to reproduce spiking behavior of the MBON- $\alpha 3$. Active channels may also be potentially distributed along the neuron's dendrites as a potential means of aiding the propagation of current from the synapse locations to the SIZ. These channels would only strengthen the depolarization at the SIZ, so the determination that stimulation of KCs can sufficiently depolarize the SIZ past a threshold is unlikely to be altered. Incorporation of these active channels would require that their conductances and dynamics be characterized in Drosophila in order for the model to be effectively parameterized. Future work can further explore associative olfactory learning in insects by augmenting the model constructed in this study to incorporate additional elements of the mushroom body such as the effect of dopaminergic feedback on the KC-MBON synapses.

\section{Materials and methods}

\subsection{Computational Model}

Morphology data for the Drosophila MBON- $\alpha 3$ was originally characterized using scanning electron microscopy [45] and for the present study obtained from the database NeuroMorpho.org [1]. These data describe the structure of the its dendritic arborization throughout the mushroom body output neuron $\alpha 3$ compartment (Figure 1B, S1A). The reconstruction is limited to the portion of the neuron in the mushroom body, not including its soma and axon.

Electrophysiological properties of the neuron were determined through a literature search, as noted below. In the absence of data specific to the Drosophila MBON- $\alpha 3$ neuron, electrophysiological parameters were chosen based on the properties of MBON neurons in other organisms (e.g., locusts) considered alongside values that would be typical throughout the Drosophila central nervous system (Table 2). For the question of electrotonic compactness, the resting (passive) transmembrane conductance is obviously crucial. We were not able to find data that reported its value explicitly for MBON neurons. Therefore we estimated its value from two sources. One is the measured dendritic time constant of $\beta$-Lobe Neurons $(\beta \mathrm{LNs})$ recorded by Cassenaer and Laurent [8]; their Figure 1b. Note however that (a) this is a different cell class (not $\alpha$-MBONs) (b) in a different insect (locust not Drosophila), that (c) the set of recorded neurons is small $(N=9)$ and that $(\mathrm{d})$ the value is an average but the variation around this average is substantial. Despite all these limitations, the values chosen are in a physiologically plausible range and we feel that they are the best estimate we can use given the extant data. The other data point is the specific membrane capacitance which is assumed to take the value $C_{m} \approx 1 \mu \mathrm{F} / \mathrm{cm}^{2}$ based on the biophysics of cell membranes [33], in excellent agreement with measured values [17]. 
All simulations were performed using the NEURON 7.6.2 simulation environment[20] running through a Python 2.7.15 interface in Ubuntu 18.04.1. The morphology data divided the MBON- $\alpha 3$ dendritic tree into 4361 small sections averaging $1.03 \mu \mathrm{m}$ in length and $0.29 \mu \mathrm{m}$ in diameter. Each section was automatically assigned an index number by NEURON. Each of these sections was modeled as a piece of cylindrical cable, with connectivity specified by the neuron's morphology. Passive leak channels were inserted along the membrane of the dendrite sections (Table 2). To determine electrotonic compactness, current was injected into selected dendritic compartments and their effect at the proximal neurite studied. Resting potential is always assumed as $-60 \mathrm{mV}$. Membrane potential data was analysed and plotted with Matlab (MathWorks) and Prism (GraphPad).

\subsection{Immunohistochemistry}

Flies were reared on standard fly food at $25^{\circ} \mathrm{C}$ and $65 \%$ humidity. Male flies expressing MB082C-Gal4 [2] and 10xUAS-mCD8GFP (BDSC 32186 [38]) were fixed for $3.5 \mathrm{~h}$ at $4^{\circ} \mathrm{C}$ in $4 \%$ PFA containing PBST $(0.2 \%$ Triton-X100). Flies were then washed for at least $3 \times 30$ min at RT in PBST before dissection. Dissected brains were then labelled with primary antibodies (rabbit anti-GFP (A6455, Life technologies, 1:2000) and mouse anti-Brp nc82, Developmental Studies Hybridoma Bank, Iowa, 1:200) for two nights at $4^{\circ} \mathrm{C}$. After incubation, brains were washed at least $6 \times 30 \mathrm{~min}$ in PBST. Secondary antibodies (Alexa488 (goat $\alpha$ rabbit) and Alexa568 (goat $\alpha$ mouse) coupled antibodies, Life technologies, 1:1000) were applied for two nights at $4^{\circ} \mathrm{C}$. After a repeated washing period, brains were submerged in Vectashield (Vector Laboratories), mounted onto microscope slides and stored at $4^{\circ} \mathrm{C}$.

Images were acquired using a confocal scanning microscope (Zeiss LSM 710) and a 25x (Zeiss PlanNEOFLUAR, NA 0.8 Korr DIC) oil objective. Raw images were processed with Fiji [41] and Adobe Photoshop. Uniform adjustments of brightness and contrast were performed in fiji and Adobe Photoshop.

\section{Acknowledgments}

Work supported by NSF 1835202, NIH R01DA040990 and NIH R01EY027544. 


\section{References}

[1] Giorgio A Ascoli, Duncan E Donohue, and Maryam Halavi. Neuromorpho. org: a central resource for neuronal morphologies. Journal of Neuroscience, 27(35):9247-9251, 2007.

[2] Yoshinori Aso, Daisuke Hattori, Yang Yu, Rebecca M Johnston, Nirmala A Iyer, Teri-TB Ngo, Heather Dionne, L F Abbott, Richard Axel, Hiromu Tanimoto, and Gerald M Rubin. The neuronal architecture of the mushroom body provides a logic for associative learning. eLife, 3:e10806, December 2014.

[3] Yoshinori Aso, Divya Sitaraman, Toshiharu Ichinose, Karla R Kaun, Katrin Vogt, Ghislain Belliart-Guérin, Pierre-Yves Plaçais, Alice A Robie, Nobuhiro Yamagata, Christopher Schnaitmann, William J Rowell, Rebecca M Johnston, Teri-T B Ngo, Nan Chen, Wyatt Korff, Michael N Nitabach, Ulrike Heberlein, Thomas Preat, Kristin M Branson, Hiromu Tanimoto, and Gerald M Rubin. Mushroom body output neurons encode valence and guide memory-based action selection in Drosophila. eLife, 3:363, December 2014.

[4] W. Becker. Metrics, volume 3 of Reviews of Oculomotor Research, chapter 2, pages 13-68. Elsevier, Amsterdam-New York-Oxford, 1989.

[5] Sandra D. Berger and Sharon M. Crook. Modeling the influence of ion channels on neuron dynamics in drosophila. Frontiers in Computational Neuroscience, 9:139, 2015.

[6] Christopher J Burke, Wolf Huetteroth, David Owald, Emmanuel Perisse, Michael J Krashes, Gaurav Das, Daryl Gohl, Marion Silies, Sarah Certel, and Scott Waddell. Layered reward signalling through octopamine and dopamine in Drosophila. Nature, 492(7429):433-437, October 2012.

[7] Andrew Campbell, Rachel Skvirsky, Henry Wortis, Sheila Thomas Ichiro Kawachi, and Christine Hohmann. Views From The Trainees - Talking About What Matters in Efforts to Diversify the STEM Workforce. CBE Life Science Education, 13:587-592, 2014.

[8] Stijn Cassenaer and Gilles Laurent. Hebbian stdp in mushroom bodies facilitates the synchronous flow of olfactory information in locusts. Nature, 448(7154):709, 2007.

[9] Paola Cognigni, Johannes Felsenberg, and Scott Waddell. Do the right thing: neural network mechanisms of memory formation, expression and update in Drosophila. Current opinion in neurobiology, 49:51-58, April 2018.

[10] Raphael Cohn, Ianessa Morantte, and Vanessa Ruta. Coordinated and Compartmentalized Neuromodulation Shapes Sensory Processing in Drosophila. Cell, 163(7):1742-1755, December 2015.

[11] J R Crittenden, E M Skoulakis, K A Han, D Kalderon, and R L Davis. Tripartite mushroom body architecture revealed by antigenic markers. Learning $\mathcal{E}$ memory (Cold Spring Harbor, N.Y.), 5(1-2):38-51, May 1998.

[12] J de Belle and M Heisenberg. Associative odor learning in Drosophila abolished by chemical ablation of mushroom bodies. Science, 263(5147):692-695, February 1994.

[13] Georg Dietzl, Doris Chen, Frank Schnorrer, Kuan-Chung Su, Yulia Barinova, Michaela Fellner, Beate Gasser, Kaolin Kinsey, Silvia Oppel, Susanne Scheiblauer, Africa Couto, Vincent Marra, Krystyna Keleman, and Barry J Dickson. A genome-wide transgenic RNAi library for conditional gene inactivation in Drosophila. Nature, 448(7150):151-156, July 2007.

[14] Katharina Eichler, Feng Li, Ashok Litwin-Kumar, Youngser Park, Ingrid Andrade, Casey M Schneider-Mizell, Timo Saumweber, Annina Huser, Claire Eschbach, Bertram Gerber, Richard D Fetter, James W Truman, Carey E Priebe, L F Abbott, Andreas S Thum, Marta Zlatic, and Albert Cardona. The complete connectome of a learning and memory centre in an insect brain. Nature, 548(7666):175-182, August 2017.

[15] John C Fiala, Josef Spacek, and Kristen M Harris. Dendrite structure. In Greg Stuart, Nelson Spruston, and Michael Häusser, editors, Dendrites, chapter 1. Oxford University Press, New York, 2007.

[16] Florence Friggi-Grelin, H 1 ne Coulom, Margaret Meller, Delphine Gomez, Jay Hirsh, and Serge Birman. Targeted gene expression inDrosophila dopaminergic cells using regulatory sequences from tyrosine hydroxylase. Journal of Neurobiology, 54(4):618-627, January 2003. 
[17] Luc J Gentet, Greg J Stuart, and John D Clements. Direct measurement of specific membrane capacitance in neurons. Biophysical journal, 79(1):314-320, 2000.

[18] Nathan W Gouwens and Rachel I Wilson. Signal propagation in drosophila central neurons. Journal of Neuroscience, 29(19):6239-6249, 2009.

[19] Annie Handler, Thomas G W Graham, Raphael Cohn, Ianessa Morantte, Andrew F Siliciano, Jianzhi Zeng, Yulong Li, and Vanessa Ruta. Distinct Dopamine Receptor Pathways Underlie the Temporal Sensitivity of Associative Learning. Cell, 178(1):60-75.e19, June 2019.

[20] M. L. Hines and N. T. Carnevale. The Neuron Simulation Environment. Neural Computation, 9(6), Aug. 1997.

[21] K S Honegger, R A A Campbell, and G C Turner. Cellular-Resolution Population Imaging Reveals Robust Sparse Coding in the Drosophila Mushroom Body. Journal of Neuroscience, 31(33):1177211785, August 2011.

[22] Cheng Huang, Pengzhi Wang, Zhiyong Xie, Lianzhang Wang, and Yi Zhong. The differential requirement of mushroom body $\alpha / \beta$ subdivisions in long-term memory retrieval in drosophila. Protein \& Cell, 4(7):512-519, 2013.

[23] Arnim Jenett, Gerald M Rubin, Teri-T B Ngo, David Shepherd, Christine Murphy, Heather Dionne, Barret D Pfeiffer, Amanda Cavallaro, Donald Hall, Jennifer Jeter, Nirmala Iyer, Dona Fetter, Joanna H Hausenfluck, Hanchuan Peng, Eric T Trautman, Robert R Svirskas, Eugene W Myers, Zbigniew R Iwinski, Yoshinori Aso, Gina M DePasquale, Adrianne Enos, Phuson Hulamm, Shing Chun Benny Lam, Hsing-Hsi Li, Todd R Laverty, Fuhui Long, Lei Qu, Sean D Murphy, Konrad Rokicki, Todd Safford, Kshiti Shaw, Julie H Simpson, Allison Sowell, Susana Tae, Yang Yu, and Christopher T Zugates. A GAL4-Driver Line Resource for Drosophila Neurobiology. Cell reports, 2(4):991-1001, October 2012.

[24] Colleen Kirkhart and Kristin Scott. Gustatory Learning and Processing in the DrosophilaMushroom Bodies. Journal of Neuroscience, 35(15):5950-5958, April 2015.

[25] Nathan C Klapoetke, Yasunobu Murata, Sung Soo Kim, Stefan R Pulver, Amanda Birdsey-Benson, Yong Ku Cho, Tania K Morimoto, Amy S Chuong, Eric J Carpenter, Zhijian Tian, Jun Wang, Yinlong Xie, Zhixiang Yan, Yong Zhang, Brian Y Chow, Barbara Surek, Michael Melkonian, Vivek Jayaraman, Martha Constantine-Paton, Gane Ka-Shu Wong, and Edward S Boyden. Independent optical excitation of distinct neural populations. Nature Methods, 11(3):338-346, February 2014.

[26] Mitsuhiko Kurusu, Takeshi Awasaki, Liria M Masuda-Nakagawa, Hiroshi Kawauchi, Kei Ito, and Katsuo Furukubo-Tokunaga. Embryonic and larval development of the Drosophila mushroom bodies: concentric layer subdivisions and the role of fasciclin II. Development, 129(2):409-419, January 2002.

[27] Sen-Lin Lai and Tzumin Lee. Genetic mosaic with dual binary transcriptional systems in Drosophila. Nature Neuroscience, 9(5):703-709, April 2006.

[28] T Lee, A Lee, and L Luo. Development of the Drosophila mushroom bodies: sequential generation of three distinct types of neurons from a neuroblast. Development, 126(18):4065-4076, September 1999.

[29] Hui-Hao Lin, Jason Sih-Yu Lai, An-Lun Chin, Yung-Chang Chen, and Ann-Shyn Chiang. A Map of Olfactory Representation in the Drosophila Mushroom Body. Cell, 128(6):1205-1217, March 2007.

[30] Lindsey J Macpherson, Emanuela E Zaharieva, Patrick J Kearney, Michael H Alpert, Tzu-Yang Lin, Zeynep Turan, Chi-Hon Lee, and Marco Gallio. Dynamic labelling of neural connections in multiple colours by trans-synaptic fluorescence complementation. Nature communications, 6(1):501, December 2015.

[31] P Masek and K Scott. Limited taste discrimination in Drosophila. Proceedings of the National Academy of Sciences, 107(33):14833-14838, August 2010.

[32] Sean M J McBride, Giovanna Giuliani, Catherine Choi, Paul Krause, Dana Correale, Karli Watson, Glenn Baker, and Kathleen K Siwicki. Mushroom Body Ablation Impairs Short-Term Memory and Long-Term Memory of Courtship Conditioning in Drosophila melanogaster. Neuron, 24(4):967-977, December 1999. 
[33] E. Niebur. Electrical properties of cell membranes. Scholarpedia, 3(6):7166, 2008.

[34] E. Niebur. Neuronal Cable Theory. Scholarpedia, 3(5):2674, 2008.

[35] David Owald, Johannes Felsenberg, Clifford B Talbot, Gaurav Das, Emmanuel Perisse, Wolf Huetteroth, and Scott Waddell. Activity of Defined Mushroom Body Output Neurons Underlies Learned Olfactory Behavior in Drosophila. Neuron, 86(2):417-427, April 2015.

[36] David Owald, Suewei Lin, and Scott Waddell. Light, heat, action: neural control of fruit fly behaviour. Philosophical Transactions of the Royal Society B: Biological Sciences, 370(1677):20140211, September 2015.

[37] A Pascual. Localization of Long-Term Memory Within the Drosophila Mushroom Body. Science, 294(5544):1115-1117, November 2001.

[38] Barret D Pfeiffer, Teri-T B Ngo, Karen L Hibbard, Christine Murphy, Arnim Jenett, James W Truman, and Gerald M Rubin. Refinement of Tools for Targeted Gene Expression in Drosophila. Genetics, 186(2):735-755, October 2010.

[39] Christopher J Potter, Bosiljka Tasic, Emilie V Russler, Liang Liang, and Liqun Luo. The Q System: A Repressible Binary System for Transgene Expression, Lineage Tracing, and Mosaic Analysis. Cell, 141(3):536-548, April 2010.

[40] W G Quinn, W A Harris, and S Benzer. Conditioned Behavior in Drosophila melanogaster. Proceedings of the National Academy of Sciences, 71(3):708-712, March 1974.

[41] Johannes Schindelin, Ignacio Arganda-Carreras, Erwin Frise, Verena Kaynig, Mark Longair, Tobias Pietzsch, Stephan Preibisch, Curtis Rueden, Stephan Saalfeld, Benjamin Schmid, Jean-Yves Tinevez, Daniel James White, Volker Hartenstein, Kevin Eliceiri, Pavel Tomancak, and Albert Cardona. Fiji: an open-source platform for biological-image analysis. Nature Methods, 9(7):676-682, June 2012.

[42] Julien Séjourné, Pierre-Yves Plaçais, Yoshinori Aso, Igor Siwanowicz, Séverine Trannoy, Vladimiros Thoma, Stevanus R Tedjakumala, Gerald M Rubin, Paul Tchenio, Kei Ito, Guillaume Isabel, Hiromu Tanimoto, and Thomas Preat. Mushroom body efferent neurons responsible for aversive olfactory memory retrieval in Drosophila. Nature Neuroscience, 14(7):903-910, June 2011.

[43] Dominique Siegenthaler, Benjamin Escribano, Vanessa Bräuler, and Jan Pielage. Selective suppression and recall of long-term memories in Drosophila. PLoS Biology, 17(8):e3000400, August 2019.

[44] Sean T Sweeney, Kendal Broadie, John Keane, Heiner Niemann, and Cahir J O'Kane. Targeted expression of tetanus toxin light chain in Drosophila specifically eliminates synaptic transmission and causes behavioral defects. Neuron, 14(2):341-351, February 1995.

[45] Shin-ya Takemura, Yoshinori Aso, Toshihide Hige, Allan Wong, Zhiyuan Lu, C Shan Xu, Patricia K Rivlin, Harald Hess, Ting Zhao, Toufiq Parag, et al. A connectome of a learning and memory center in the adult Drosophila brain. Elife, 6:e26975, 2017.

[46] Nobuaki K Tanaka, Hiromu Tanimoto, and KEI Ito. Neuronal assemblies of the drosophila mushroom body. Journal of Comparative Neurology, 508(5):711-755, 2008.

[47] Gerhard M Technau. Fiber Number in the Mushroom Bodies of Adult Drosophila melanogasterdepends on Age, Sex and Experience. Journal of neurogenetics, 1(2):113-126, July 2009.

[48] Lin Tian, S Andrew Hires, Tianyi Mao, Daniel Huber, M Eugenia Chiappe, Sreekanth H Chalasani, Leopoldo Petreanu, Jasper Akerboom, Sean A McKinney, Eric R Schreiter, Cornelia I Bargmann, Vivek Jayaraman, Karel Svoboda, and Loren L Looger. Imaging neural activity in worms, flies and mice with improved GCaMP calcium indicators. Nature Methods, 6(12):875-881, November 2009.

[49] Tim Tully and William G Quinn. Classical conditioning and retention in normal and mutantDrosophila melanogaster. Journal of Comparative Physiology A, 157(2):263-277, March 1985.

[50] Glenn C Turner, Maxim Bazhenov, and Gilles Laurent. Olfactory Representations by DrosophilaMushroom Body Neurons. Journal of neurophysiology, 99(2):734-746, February 2008.

[51] Katrin Vogt, Christopher Schnaitmann, Kristina V Dylla, Stephan Knapek, Yoshinori Aso, Gerald M Rubin, and Hiromu Tanimoto. Shared mushroom body circuits underlie visual and olfactory memories in Drosophila. eLife, 3:156, August 2014. 
[52] Leslie B Vosshall, Hubert Amrein, Pavel S Morozov, Andrey Rzhetsky, and Richard Axel. A Spatial Map of Olfactory Receptor Expression in the Drosophila Antenna. Cell, 96(5):725-736, March 1999.

[53] Zhihao Zheng, J Scott Lauritzen, Eric Perlman, Camenzind G Robinson, Matthew Nichols, Daniel Milkie, Omar Torrens, John Price, Corey B Fisher, Nadiya Sharifi, Steven A Calle-Schuler, Lucia Kmecova, Iqbal J Ali, Bill Karsh, Eric T Trautman, John A Bogovic, Philipp Hanslovsky, Gregory S X E Jefferis, Michael Kazhdan, Khaled Khairy, Stephan Saalfeld, Richard D Fetter, and Davi D Bock. A Complete Electron Microscopy Volume of the Brain of Adult Drosophila melanogaster. Cell, 174(3):730-743.e22, July 2018. 


\section{Supplementary Material}

\section{A}

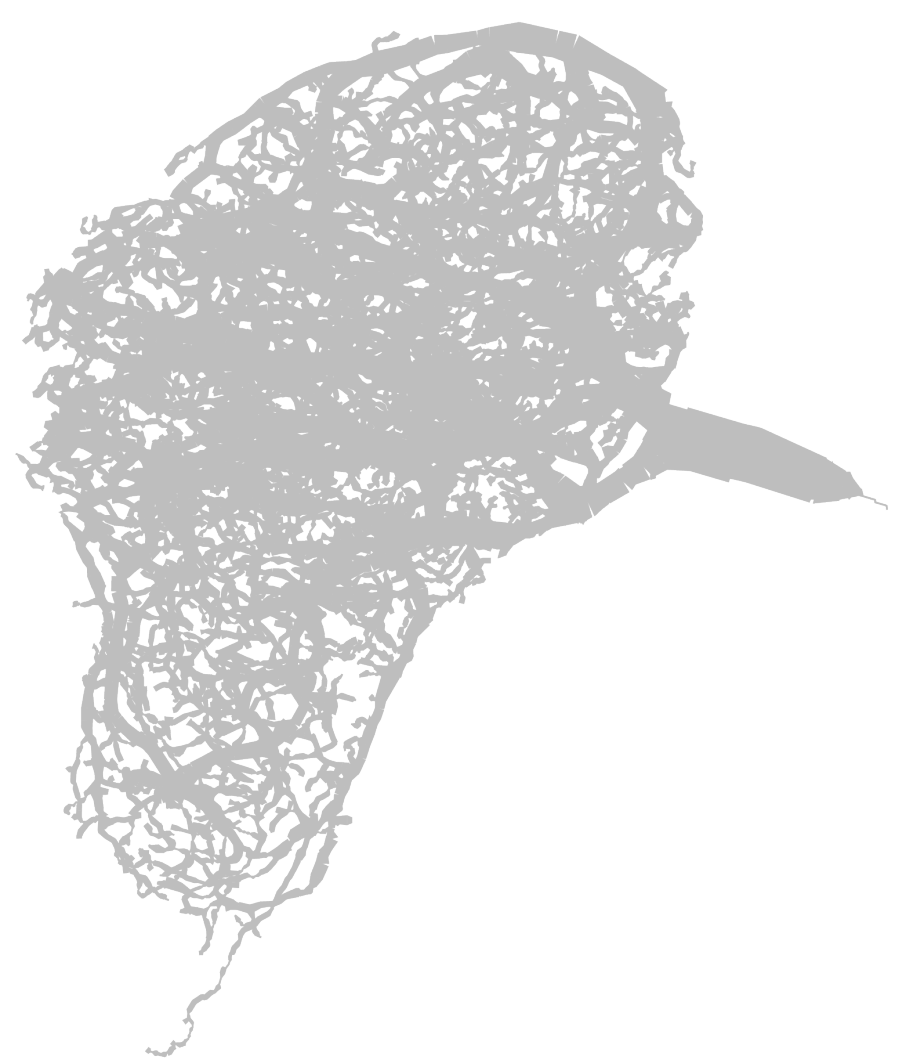

B

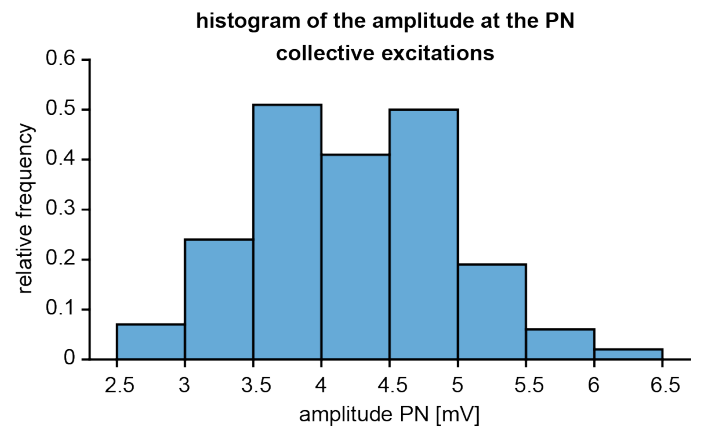

C

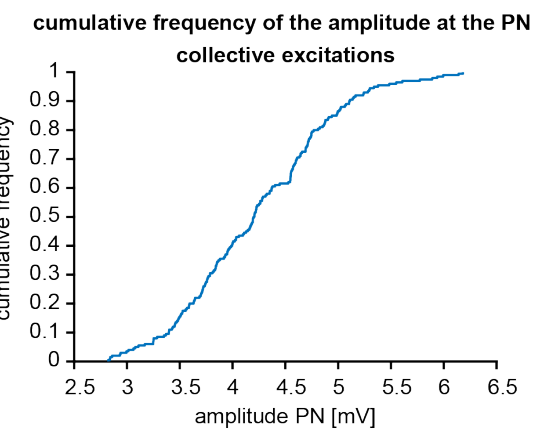

Figure S1: Projection of dendritic morphology and statistics of the amplitudes from collective excitations. Amplitudes were measured through 200 simulation runs of collective excitation patterns, each consisting of current injection into 25 randomly selected dendritic segments. (A) Projection of the complete dendritic reconstruction morphology. (B) Relative frequency distribution of the amplitude from dendritic stimulation for all 200 excitation patterns. Bin width $0.5 \mathrm{mV}$. (C) Same, but shown is the cumulative frequency distribution. 
A

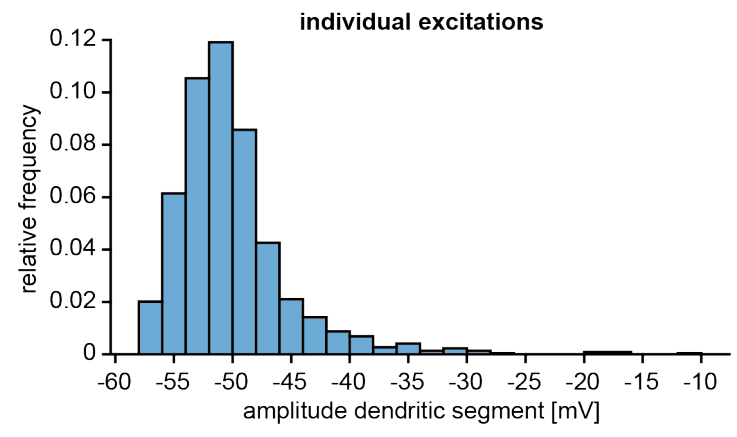

C

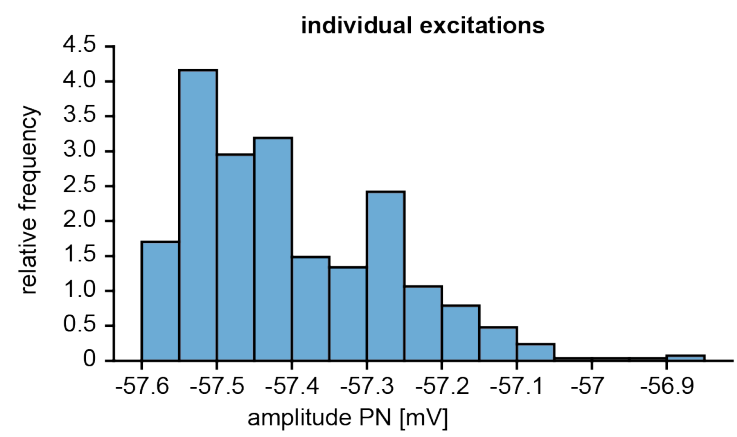

E

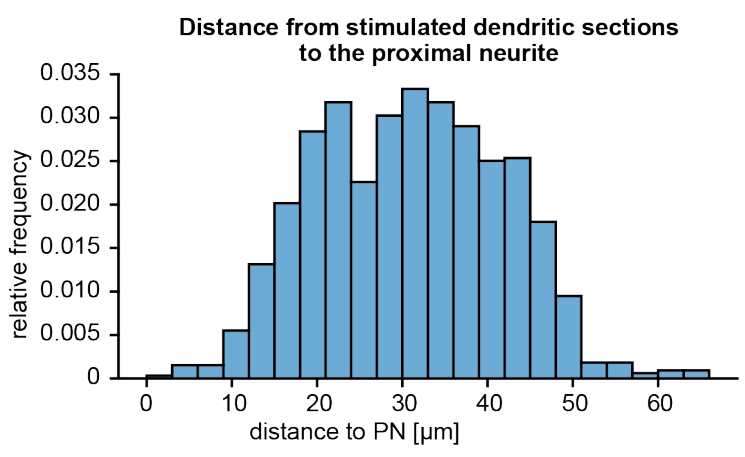

B

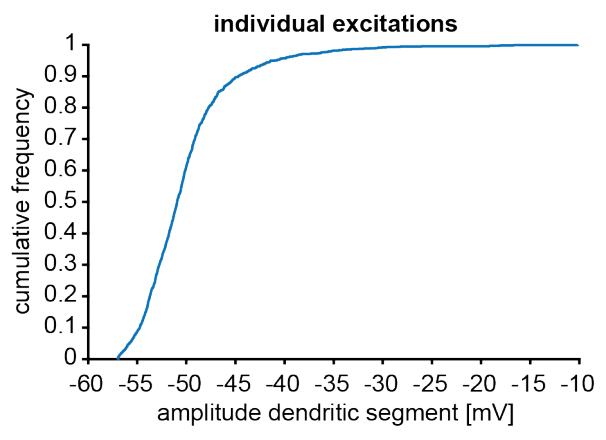

D

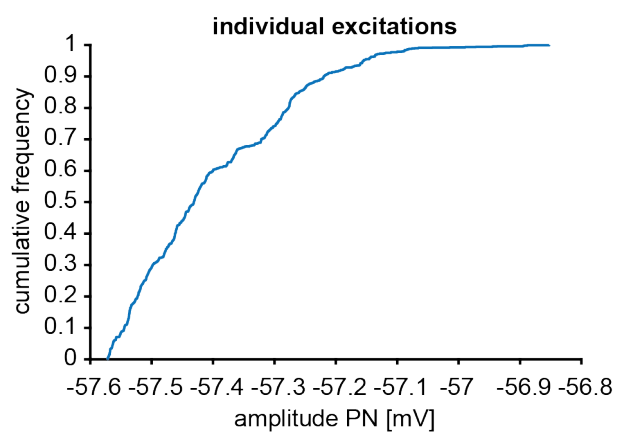

$\mathbf{F}$

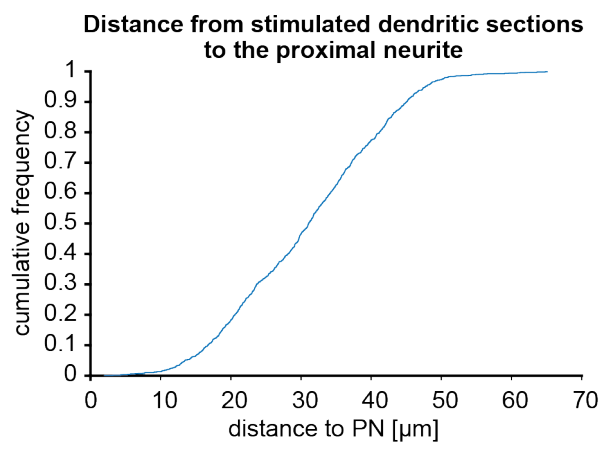

Figure S2: Distribution of maximal voltages in dendritic tree and proximal neurite, demonstrating the electrotonic compactness of the dendritic tree. Voltage excursions are responses to the injection of identical currents in 1091 randomly selected segments in the dendritic tree (A) Relative frequency distribution of maximal voltage amplitude in stimulated dendritic segments. Bin width $2 m V$. (B) Same, but shown as cumulative frequency distribution. (C) Same as (A) but measured in the proximal neurite. Bin width $0.05 \mathrm{mV}$. (D) Same as (B), but measured in the proximal neurite. (E) Relative frequency of the distance along the dendrite of a stimulated dendritic segment from the proximal neurite. Bin width $3 \mu m$ (F) Same, but shown is the cumulative frequency distribution. 